\title{
Facile synthesis of reduced graphene oxide/ peroxomolybdate(VI)-citrate composite and its potential energy storage application
}

\author{
Mateusz Ciszewski $^{1}$ - Grzegorz Benke ${ }^{1} \cdot$ Katarzyna Leszczyńska-Sejda $^{1}$ \\ Dorota Kopyto ${ }^{1}$
}

Received: 17 July 2017 / Accepted: 14 October 2017 / Published online: 23 October 2017

(C) The Author(s) 2017. This article is an open access publication

\begin{abstract}
A new energy storage material based on molybdate active species has been presented. Molybdenum seems to be a perspective material in supercapacitors because of numerous possible metal oxidation states, electrolyte storage by means of various chemical reactions and availability in comparison to other refractory metals. Material synthesized within this research was composed of reduced graphene oxide matrix and peroxomolybdate(VI)-citrate active dimers. It was showed that peroxomolybdate(VI)-citrate structure enhanced electrochemical activity of symmetric supercapacitor. Simple methodology was used to synthesize a composite with $\mathrm{pH}$ adjustment as the key step. The specific capacity calculated from galvanostatic charge/discharge curves was as high as $250 \mathrm{~F} / \mathrm{g}$. Material was distinguished by good cyclability with $5 \%$ capacity loss after 1000 cycles. The increase in charge transfer resistance, induced by metal-oxygen compound within the carbon matrix was relatively low, compared to parent reduced graphene oxide. Amorphous structure of peroxomolybdate(VI)-modified material was observed with slight increase in the interlayer distance in comparison to parent reduced graphene oxide. The height and lateral size of crystallites were also determined. Significant decrease in the specific surface area of peroxomolybdate(VI)-modified composite was observed, in comparison to the parent reduced graphene oxide.
\end{abstract}

Mateusz Ciszewski

mateusz.ciszewski@imn.gliwice.pl

1 Department of Hydrometallurgy, Institute of Non Ferrous Metals, Sowińskiego 5, 44-100 Gliwice, Poland

\section{Introduction}

Transition metal oxides are commonly used in energy storage materials because of relatively high increase in specific capacity through so-called pseudocapacitance, availability, in many cases low price and because of chemical and physical properties [1,2]. Among them refractory metals (molybdenum, tungsten, rhenium, tantalum and niobium) are rarely taken into account mainly because of economy and low abundance. Molybdenum, tungsten and rhenium may be recognized as potentially effective materials that are able to enhance the specific capacity electrode, which is attributed to numerous oxidation states of these metals. The chemistry of rhenium seems to be out of scope in this matter as it is very rare and expensive metal. However, molybdenum and tungsten are relatively easily available. In this paper, the possibility of molybdenum species application in energy storage materials was examined. Molybdenum has high thermal stability, high thermal and electric conductivity, is corrosion resistant and has low thermal expansion coefficient. This makes it perfect for applications such as electronics, metallurgy, aerospace industry, lighting technologies and coatings [3]. In the literature, numerous examples of molybdenum(IV) oxide and molybdenum(VI) oxide as well as molybdenum(IV) sulfide-based electrodes for supercapacitors and lithium-ion batteries can be found. The specific capacity of these materials may be varied from $36 \mathrm{~F} / \mathrm{g}$ for $\mathrm{MoO}_{2}$ to $288 \mathrm{~F} / \mathrm{g}$ for $\alpha-\mathrm{MoO}_{3}$ [4]. A perspective material is also molybdenum sulfide that may result in ca. $410 \mathrm{~F} / \mathrm{g}$ [5]. An important feature of molybdenum is capability to form a lot of various structures that are composed of molybdenum-oxygen bonds. The chemistry of molybdenum compounds is a vast range of different molybdenum-oxygen-hydrogen combinations [6] that may be perfectly suited as pseudocapacitive materials in energy storage. Another 
important class of compounds exhibiting reversible redox activity is molybdates. Their high catalytic activity, good electrochemical properties and environmental friendliness have driven them to energy storage applications [7]. The specific capacity reported for various molybdate-based supercapacitors for single electrode obtained using three electrode system was as high as $65 \mathrm{~F} / \mathrm{g}$ for $\mathrm{Ag}_{6} \mathrm{Mo}_{10} \mathrm{O}_{33}$ nanostructures [8], $170 \mathrm{~F} / \mathrm{g}$ for $\mathrm{CoMoO}_{4}$ nanospheres [9], $187 \mathrm{~F} / \mathrm{g}$ for $\mathrm{MnMoO}_{4} / \mathrm{CoMoO}_{4}$ nanowires [10], $200 \mathrm{~F} / \mathrm{g}$ for $\alpha-\mathrm{MnMoO}_{4}$ [11], $212 \mathrm{~F} / \mathrm{g}$ for carbon sphere@ $\mathrm{NiMoO}_{4}$ [12], and 974F/g for $\mathrm{NiMoO}_{4}$ nanorods and nanospheres [13]. While using two-electrode system in a symmetric configuration, $81 \mathrm{~F} / \mathrm{g}$ was obtained for a- $\mathrm{MnMoO}_{4}[11]$ and $53 \mathrm{~F} / \mathrm{g}$ for $\mathrm{MoS}_{2} / \mathrm{RGO}$ nanocomposite [14].

In lithium-ion batteries, iron molybdate and tin molybdate can be found $[15,16]$. The great attention has been recently attracted to more complex molybdate like copper hydroxy molybdate or zinc-hydroxy molybdate and their potential electrochemical activity $[17,18]$. This paper reports facile synthesis of graphene-based peroxomolybdate(VI)-citrate composite. A properly adjusted $\mathrm{pH}$ enhanced formation of peroxomolybdate(VI)-citrate dimers $\mathrm{M}_{5}\left[\mathrm{MoO}\left(\mathrm{O}_{2}\right)_{2}-(\mathrm{Hcit})\right.$ $\left.\mathrm{H}(\mathrm{Hcit})\left(\mathrm{O}_{2}\right)_{2} \mathrm{OMo}\right] \cdot 6 \mathrm{H}_{2} \mathrm{O}$ [19] that were used as pseudocapacitive active species in enhancing non-faradaic reactions.

\section{Experimental}

\subsection{Material preparation}

Graphite oxide was prepared using Hummers method [20] by mixing $10 \mathrm{~g}$ synthetic graphite with $230 \mathrm{~mL} 95-97 \%$ sulfuric acid (Acros Organics), $5 \mathrm{~g}$ sodium nitrate and $30 \mathrm{~g}$ potassium permanganate in a round-bottomed flask equipped with a reflux and thermometer and ice-cooled at the temperature close to $273 \mathrm{~K}$. After $8 \mathrm{~h}$, GO slurry was transferred diluted with deionized water, washed several times by decantation and vacuum-filtrated using two filter papers (Munktell, 390). As-prepared GO was dried at $110^{\circ} \mathrm{C}$ in an oven. Graphite oxide was then milled in a mortar and ultrasonically dispersed in water. Next, hydrazine hydrate was added, mixture was sonicated and then boiled on a hot plate. Finally it was filtered, washed with copious amount of water and dried at $110{ }^{\circ} \mathrm{C}$. Reduced graphene oxide was denoted as RGrO.

Molybdenum active species was synthesized as follows: $2.4 \mathrm{~g} \mathrm{Na}_{2} \mathrm{MoO}_{4} \cdot 2 \mathrm{H}_{2} \mathrm{O}$ was dissolved in $10 \mathrm{ml}$ water, $0.7 \mathrm{ml}$ $30 \% \mathrm{H}_{2} \mathrm{O}_{2}$ was added. Then solution containing $1.9 \mathrm{~g}$ citric acid in water was added and the mixture was thoroughly mixed.

Next, reduced graphene oxide was dispersed in $50 \mathrm{ml}$ and $\mathrm{pH}$ was adjusted by $1 \mathrm{M} \mathrm{HNO}_{3}$ to ca. 4.7. Molybdenum precursor was added to reduced graphene oxide and components were ultrasonically mixed for $15 \mathrm{~min}$ and then simultaneously magnetically mixed and boiled. Finally material was vacuum-filtrated using micro-glass filters and dried. $0.17 \mathrm{~g}$ product denoted as RedNaCA (Reduced graphene oxide Na-based molybdate and Citric Acid) was obtained.

\subsection{Characterization}

XRD analysis was performed using Co $K \alpha$ lamp in $2 \theta$ range from $10^{\circ}$ to $100^{\circ}$ (Seifert FPM) - the use of cobalt lamp instead of commonly used copper was imposed by technical problems. BET surface area was analyzed using pore structure analyzer ( $3 \mathrm{Flex}^{\mathrm{TM}}$, Micromeritics). SEM analysis was performed using JXA 8230, Jeol, to show the morphology of considered materials. Electrochemical experiments were carried out using two-electrode system presented in Fig. 1.

The working electrode materials were pasted on electrochemical nickel current collectors to form films and separated with glass microfiber membrane (Whatman) soaked with $6 \mathrm{M} \mathrm{KOH}$. The accurate weight of the electrodes was read by a high-precision balance (Mettler Toledo AB 204S). Electrodes, current collectors and separator were pressed by four screws in a poly(methyl methacrylate) (PMMA) casing. Cyclic voltammetry (CV) in $0-1 \mathrm{~V}$ window at scan rate $500 \mathrm{mV} / \mathrm{s}$, galvanostatic charge/discharge (GC) at current density in a range $0.1-6 \mathrm{~A} / \mathrm{g}$, and electrochemical impedance spectroscopy (EIS) in the frequency range $100 \mathrm{kHz}-100 \mathrm{mHz}$ with the amplitude of sinusoidal voltage signal equal to $10 \mathrm{mV}$ were performed with Autolab PGSTAT 302N workstation.

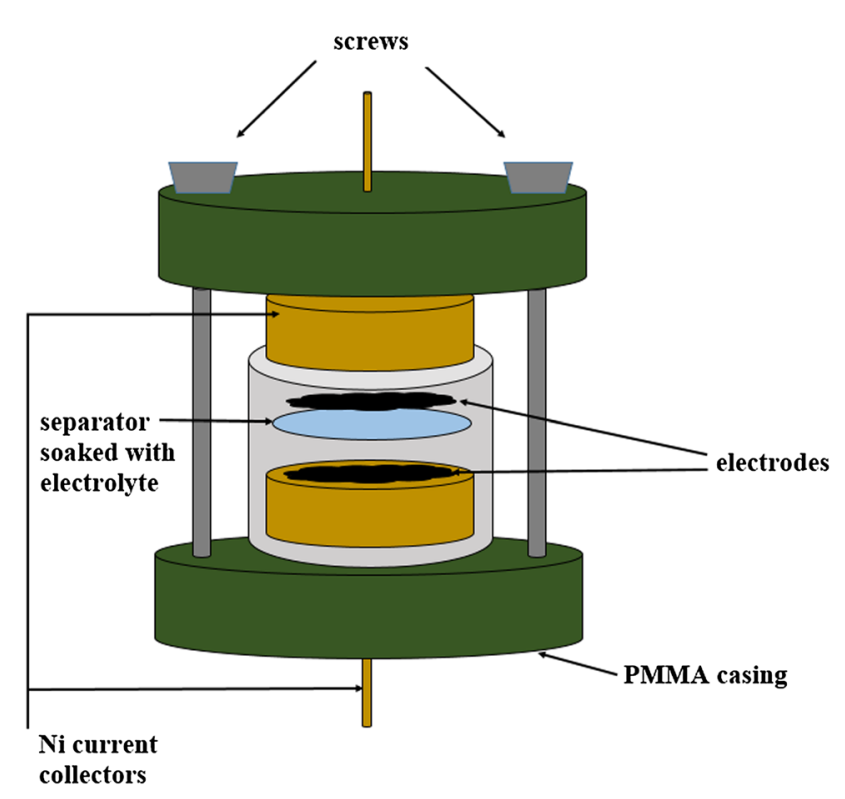

Fig. 1 Test cell for electrochemical analysis 


\section{Results}

Scheme of reduced graphene oxide/ peroxomolybdate(VI)-citrate composite preparation is presented in Fig. 2. Process was fast and easy. First, graphite oxide was ultrasonically exfoliated into graphene oxide and then chemically reduced. Second, peroxomolybdate(VI)-citrate complex was prepared by oxidation of sodium molybdate(VI) precursor with concentrated hydrogen peroxide with the addition of citric acid as complexing agent. Both solutions were ultrasonically and magnetically mixed together and boiled. Dry product was visually very similar to parent reduced graphene oxide but electrochemically much different.

XRD patterns of the peroxomolybdate composite and parent reduced graphene oxide are presented in Fig. 3. In both materials, two signals located around $27^{\circ}$ and $52^{\circ}$ were recorded. These signals were attributed to the

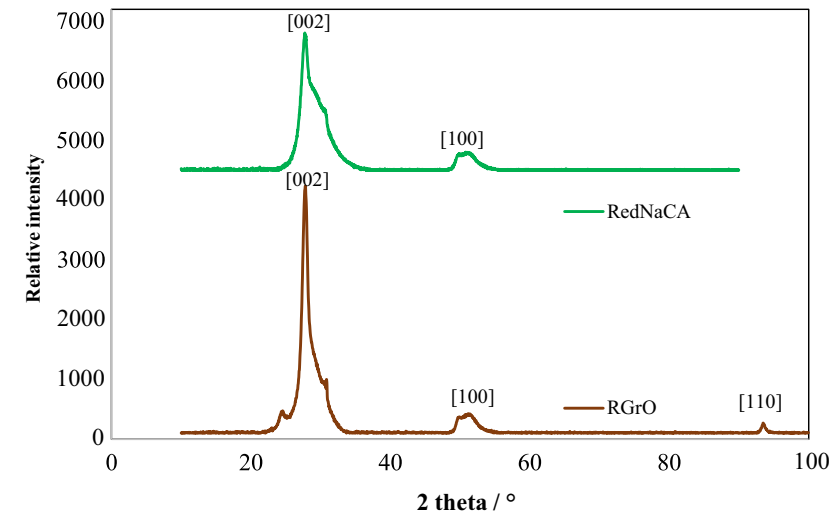

Fig. 3 XRD patterns for RGrO and RedNaCA

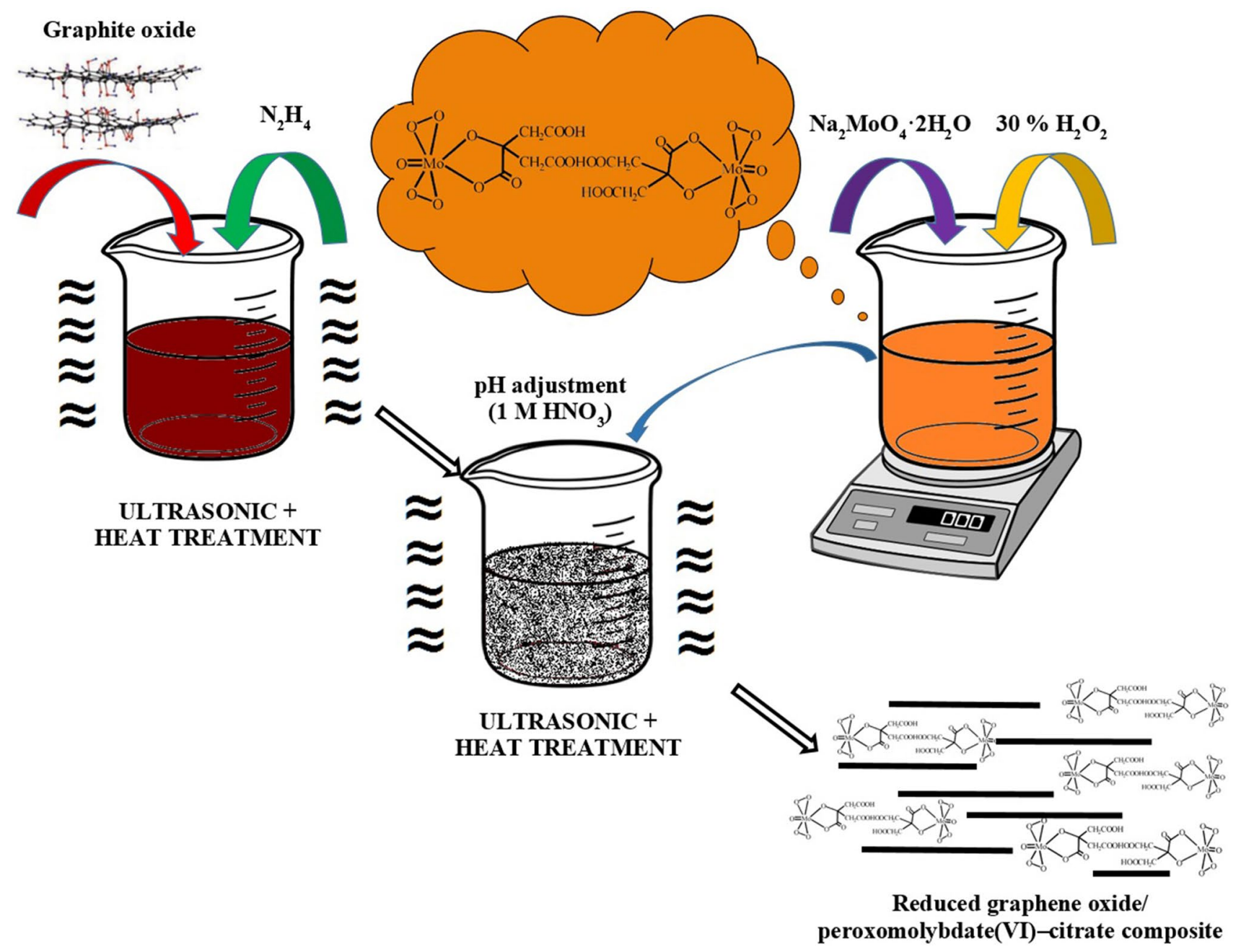

Fig. 2 Schematic presentation of reduced graphene oxide/peroxomolybdate(VI)-citrate composite preparation 
matrix - reduced graphene oxide; however, it should be emphasized that obtained $2 \theta$ angle values are bigger than commonly found in the literature. This was a result of cobalt lamp used in analysis, which caused peak shift to bigger angles with respect to copper lamp (the application of Co $K \alpha$ lamp was pursued by the Analytical Department of Institute of Non-Ferrous Metals responsible for all XRD analyses). Regardless of peak shifts, the localization of signals was appropriate with respect to references. Unfortunately, no strong molybdenum signal was detected that indicates the amorphous structure of the peroxomolybdate(VI)-citrate phase. The analysis performed using ParSize analyzer showed that the interlayer distance for $\mathrm{RGrO}$ and RedNaCA was as high as 0.35 and $0.37 \mathrm{~nm}$, respectively, while the theoretical number of graphene layers within material was 31 for RGrO and 29.3 for RedNaCA. The crystallite size $\left(L_{\mathrm{c}}-\right.$ crystallites height and $L_{\mathrm{a}}$-lateral size) of graphene domains calculated using
Sherrer equation was as high as 11.1 and $19.9 \mathrm{~nm}$ for $\mathrm{RGrO}$ and 10.5 and $18.7 \mathrm{~nm}$ for RedNaCA.

The specific surface area evaluated for both materials was $26.2 \mathrm{~m}^{2} / \mathrm{g}$ for RGrO and merely $4.4 \mathrm{~m}^{2} / \mathrm{g}$ for RedNaCA. Low surface area of the composite is in accordance with assumptions as the material was obtained in reaction of reduced graphene oxide with molybdenum precursor, which might partially clog the pores.

Morphological studies were performed using scanning electron microscopy. Results are presented in Fig. 4.

Amorphous phase of embedded peroxomolybdate-citrate complex as well as low magnification of the microscope did not allow to observe specific molybdenum particles within the graphene layer. It was found that for parent reduced graphene oxide slightly more corrugated structure was reported that may indicate clogging of interlayer spaces of RedNaCA by active material. This is in accordance with the specific surface area data.
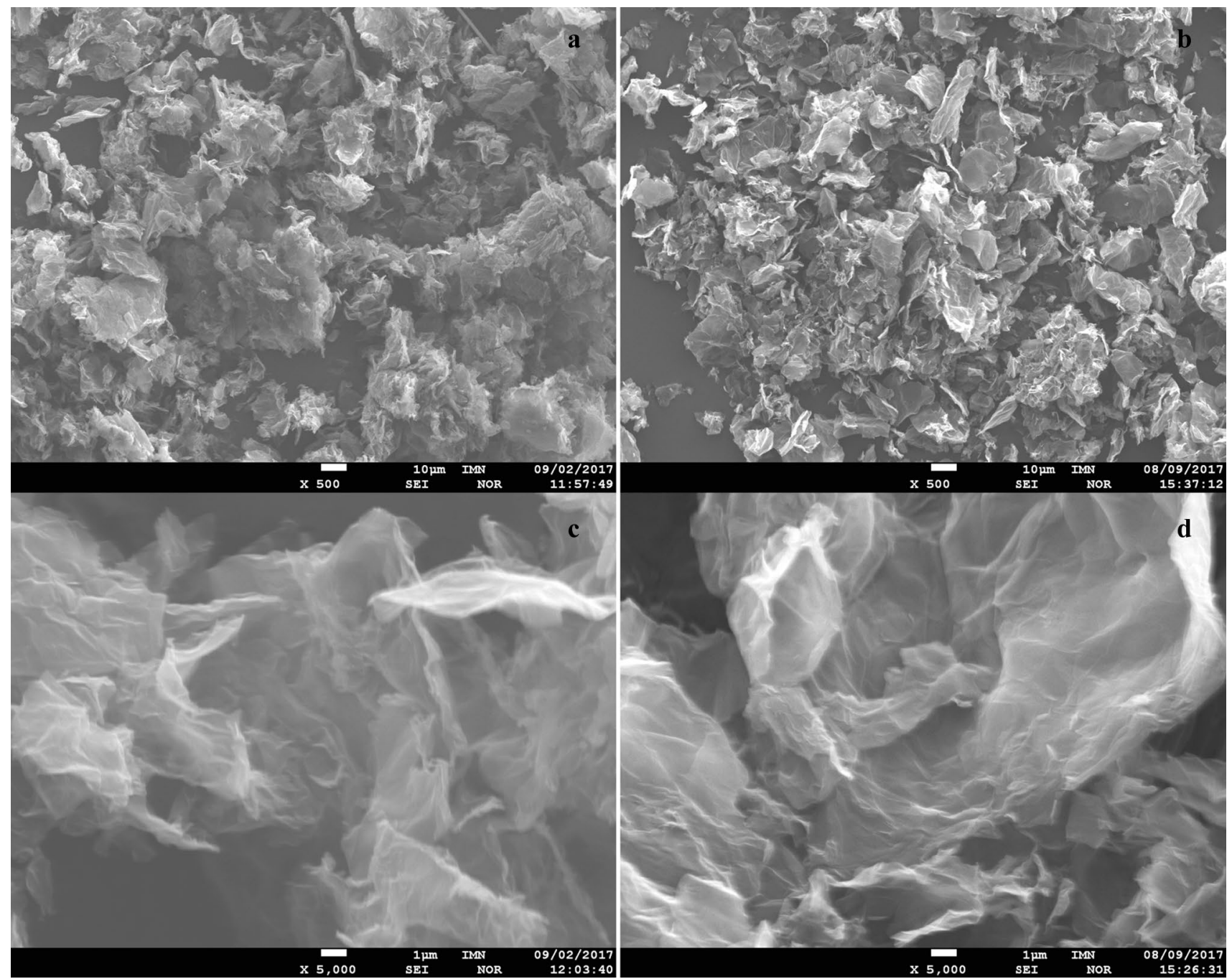

Fig. 4 SEM images recorded for $\operatorname{RedNaCA}(\mathbf{a}, \mathbf{c})$ and $\operatorname{RGrO}(\mathbf{b}, \mathbf{d})$ at magnification $\times 500$ and $\times 5000$ 
Results of the electrochemical tests performed using reduced graphene oxide and its composite with peroxomolybdate-citrate are presented in Fig. 5. The most credible method to evaluate the specific capacity of the prepared material is galvanostatic charge/discharge characteristic. The specific capacity was calculated using the following formula: $C_{\mathrm{SP}}=i \times t /(m \times \Delta V)$, where $C_{\mathrm{SP}}$ is the specific capacity, $i$ is the current intensity, $t$ is the discharge time, $m$ is the mass of active material, and $\Delta V$ is the potential difference.

Both materials were examined using current density range from 0.1 to $6 \mathrm{~A} / \mathrm{g}$. The recorded specific capacity (Fig. 5a) was as high as $147 \mathrm{~F} / \mathrm{g}$ for a parent $\mathrm{RGrO}$ and $250 \mathrm{~F} / \mathrm{g}$ for RedNaCA. Charge and discharge curves were ideal-like symmetrical; however, for RedNaCA no apex curvature was observed and material was able to discharge completely. Cyclic voltammetry was used to determine the capacity loss during 1000 cycles. Electrodes were swept with a potential at a scan rate $500 \mathrm{mV} / \mathrm{s}$ in a potential window $0-1 \mathrm{~V}$, the most common for aqueous electrolyte. Although not very much resembling box-like shape (rounded edges), CV curves were symmetric with an acceptable value of recorded current intensity (Fig. 5b). This indicates reversibility of electrode processes; however, some barriers were present that impeded charge and discharge. Slightly enhanced reactions' reversibility was reported for RedNaCA with a longer plateau region in the range $0.4-1 \mathrm{~V}$ (charging) and $0.55-0 \mathrm{~V}$ (discharging). Some shape imperfections with respect to ideal box-like shape may result from improper pore distribution of required size or some spatial obstacles within material caused by active species, which was synthesized within pores. A decrease in the specific capacity after 1000 cycles, calculated from $\mathrm{CV}$ curves, was 5 and $7 \%$ for RedNaCA and RGrO, respectively (Fig. 5c). The relation of real impedance vs imaginary impedance was presented in the form of Nyquist plot (Fig. 5d). Generally, such curves are composed of two-component semicircle over the high-frequency region corresponding to the Faradaic charge transfer resistance and linear part in a low-frequency region indicating a pure capacitive behavior and representing the ion diffusion in the electrode structure. These data allowed to estimate electrolyte resistance $R_{\mathrm{S}}$, which was 0.13 and $1.25 \Omega$ for RedNaCA and $\mathrm{RGrO}$, respectively, and charge transfer resistance $R_{\mathrm{CT}}$, 2.07 and $1.1 \Omega$, respectively. This slightly bigger value of $R_{\mathrm{CT}}$ was responsible for slower kinetics. Smaller loop and $45^{\circ}$ curve in the low-frequency region are attributed to the better Warburg diffusion caused by improved mass transfer from the electrolyte to electrode interface. As there was no significant "chemical obstacles" within RGrO the net real impedance value $(10 \Omega)$ was slightly lower in comparison to the RedNaCA $(15 \Omega)$.
Fig. 5 Results of electrochemical tests: a galvanostatic charge/ discharge (RedNaCA—green, $\mathrm{RGrO}$ - brown), b cyclic voltammetry curves at scan rate $500 \mathrm{mV} / \mathrm{s}$, c capacity loss calculated from $\mathrm{CV}$ curves and $\mathbf{d}$ Nyquist plots at frequency range $100 \mathrm{k} \Omega-100 \mathrm{~m} \Omega$
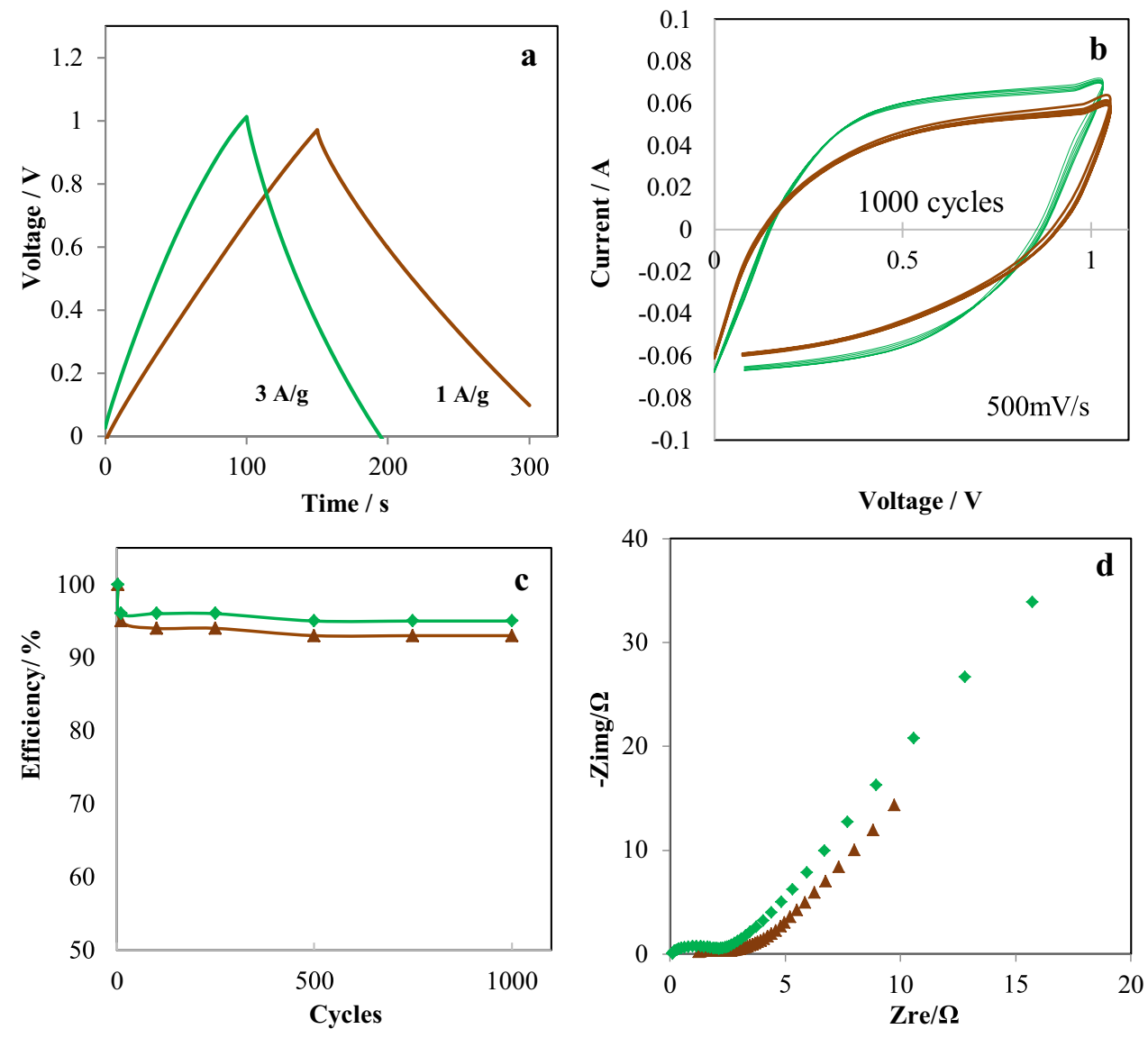


\section{Conclusions}

A new type of peroxomolybdate(VI)-citrate structure synthesized within reduced graphene oxide matrix using simple pH adjustment method was developed. It was demonstarted that peroxymolybdate(VI)-citrate species effectively enhanced specific capacity of reduced graphene oxide. Specific capacity obtained from galvanostatic charge/ discharge curves for composite material was as high as $250 \mathrm{~F} / \mathrm{g}$, i.e., about $100 \mathrm{~F} / \mathrm{g}$ more than for parent reduced graphene oxide. Cycling stability was also improved with the capacity loss after 1000 cycles at the level merely $5 \%$. Although an increase in charge transfer resistance was observed, the net impedance relation for composite material was advantageous with respect to the matrix (smaller loop and more steep curve). It was found that embedded peroxomolybdate(VI)-citrate species resulted in a decrease in specific surface area, by partial clogging of matrix pores. Although slightly increased interlayer distance was observed for the composite the theoretical number of layers as well as crystallites size was smaller in comparison to the reduced graphene oxide.

Open Access This article is distributed under the terms of the Creative Commons Attribution 4.0 International License (http://creativecommons.org/licenses/by/4.0/), which permits unrestricted use, distribution, and reproduction in any medium, provided you give appropriate credit to the original author(s) and the source, provide a link to the Creative Commons license, and indicate if changes were made.

\section{References}

1. M. Minakshi, M.J. Barmi, R.T. Jones, Dalton Trans. 46, 3588 (2017)
2. T. Watcharatharapong, M.M. Sundaram, S. Chakraborty, D. Li, G.M. Shafiullah, R.D. Aughterson, R. Ahuja, ACS Appl. Mater. Interfaces 9, 17977 (2017)

3. Z. Bao, X. Xiaomei, L. Qian, Rare Met. 31, 517 (2012)

4. J. Zhou, J. Song, H. Li, X. Feng, Z. Huang, S. Chen, Y. Ma, L. Wang, X. Yan, New J. Chem. 39,8780 (2015)

5. T. Sun, Z. Li, X. Liu, L. Ma, J. Wang, S. Yang, J. Power Sources 331, 180 (2016)

6. K.-H. Tytko, W.-D. Fleischmann, D. Gras, E. Warkentin, Mo Molybdenum, Hydrous Molybdates of Groups VA to VIS Metals (Springer, Berlin, 1985), pp. 1-271

7. J. Haetge, C. Suchomski, T. Brezesinski, Small 9, 2541 (2013)

8. V. Kumar, S. Matz, D. Hoogestraat, V. Bhavanasi, K. Parida, K. Al-Shamery, P.S. Lee, Adv. Mater. 28, 6966 (2016)

9. M. Jozegholami, M.M. Sundaram, RSC Adv. 6, 36152 (2016)

10. B.L.-Q. Mai, F. Yang, Y.-L. Zhao, X. Xu, L. Xu, Y.-Z. Luo, Nat. Commun. 2, 1 (2011)

11. B. Senthilkumar, R. K.Selvan, D. Meyrick, M. Minakshi, Int. J. Electrochem. Sci. 10, 185 (2015)

12. C. Wei, Y. Huang, J. Yan, X. Chen, X. Zhang, Ceram. Int. 42, 15694 (2016)

13. D. Cai, D. Wang, Bin Liu, Y. Wang, Y. Liu, L. Wang, H. Li, H. Huang, Q. Li, T. Wang, ACS Appl. Mater. Interfaces 5, 12905 (2013)

14. Y. Zhang, P. Ju, C. Zhao, X. Qian, Electrochim. Acta 219, 693 (2016)

15. Z. Ju, E. Zhang, Y. Zhao, Z. Xing, Q. Zhuang, Y. Oiang, Y. Qian, Small 11, 4753 (2015)

16. S.R. Ede, V. Mani, N. Kalaiselvi, S. Kundu, New J. Chem. 40, 6185 (2016)

17. B. Swain, D.H. Lee, J.R. Park, C.G. Lee, K.J. Lee, D.W. Kim, K.S. Park, Cryst. Eng. Commun. 19, 154 (2017)

18. J. Xia, L.X. Song, W. Liu, Y. Teng, Q.S. Wang, L. Zhao, M.M. Ruan, RSC Adv. 5, 12015 (2015)

19. Z.-H. Zhou, S.-Y. Hou, H.-L. Wan, Dalton Trans. 7, 1393 (2004)

20. W.S. Hummers, R.E. Offeman, J. Am. Chem. Soc. 80, 1339 (1958) 